\title{
Comparative numerical investigation of Burgers' equation with and without Hopf-Cole transformation
}

\author{
Mayur P. Bonkile
}

Department of Mechanical Engineering, National Institute of Technology Calicut, Kozhikode pin: 673 601, Kerala, India Email: bonkile.mayur@gmail.com

*Corresponding author

\section{Ashish Awasthi}

Department of Mathematics,

National Institute of Technology Calicut, Kozhikode pin: 673 601, Kerala, India

Email: aawasthi@nitc.ac.in

\section{S. Jayaraj}

Department of Mechanical Engineering, National Institute of Technology Calicut, Kozhikode pin: 673 601, Kerala, India Email: sjayaraj@nitc.ac.in

\begin{abstract}
Even if the numerical simulation of the unsteady viscous Burgers' equation is well documented in the literature, a detailed literature survey indicates that there is still gaps exists for comparative investigation regarding the effect of Hopf-Cole transformation on the efficiency and accuracy of schemes. In this paper, a comparative numerical investigation of Burgers' equation is presented based on two different approaches. Hopf-Cole transformation is implemented on this equation and then solved by modified Keller box scheme. We sketch a new implicit scheme with second order accuracy in space and time, which is proposed to solve Burgers' equation without using Hopf-Cole transformation. Numerical results of two test problems, which are calculated for various values of kinematic viscosity and time steps, are found to be matching with the exact solution. The new implicit box scheme is proved to be more accurate than the modified Keller box scheme with Hopf-Cole transformation based on $L_{2}$ and $L_{\infty}$ errors.
\end{abstract}

Keywords: finite difference method; Burgers' equation; Hopf-Cole transformation.

Reference to this paper should be made as follows: Bonkile, M.P., Awasthi, A and Jayaraj, S. (2016) 'Comparative numerical investigation of Burgers' equation with and without Hopf-Cole transformation', Int. J. Convergence Computing, Vol. 2, No. 1, pp.54-78. 
Biographical notes: Mayur P. Bonkile received his BTech in Mechanical Engineering from Government College of Engineering Amravati (2011) and MTech in Thermal Sciences from National Institute of Technology Calicut (2015), India. Currently, he is a Research Scholar at Department of Energy Science and Engineering, Indian Institute of Technology Bombay, India. His main research directions are computational analysis of fluid flow and heat transfer, numerical methods, modelling-simulation and optimisation of electrochemical energy storage systems.

Ashish Awasthi received his BSc degree from CSJM University Kanpur, India in 1997 and MSc in Mathematics from CSJM University Kanpur in 1999. He completed his PhD in Mathematics from the Department of Mathematics, Indian Institute of Technology Kanpur in 2007. He worked as an Assistant Professor in the Department of Mathematics, NIT Warangal from Jan.2007 to June 2008. He joined Upper Austria University of Applied Sciences as a Post-Doc Fellow in 2008. He worked on European Union Project ICESTARS. He joined NIT Calicut as an Assistant Professor in 2010. He is working in the area of computational sciences, numerical methods for solving ODEs and PDEs and singular perturbation theories for differential equations. He has published several papers in international journals.

S. Jayaraj received his MTech from Indian Institute of Technology, Madras (1980) and PhD from Indian Institute of Technology, Kanpur (1988). During 2005-06, he was a Post-Doctoral Fellow at the Dong-A University, Republic of Korea. Currently, he is a Professor at the Department of Mechanical Engineering, National Institute of Technology Calicut, India. He has 35 years of teaching experience during which he has guided nine $\mathrm{PhD}$ and 65 MTech students. He is having visiting faculty status at the Kazakh National University, Almaty, Kazakhstan. He is a well-known name in solar-thermal scientific community for his research. He has co-authored more than 100 peer reviewed international journal papers and 150 papers got published in proceedings of international conferences. Also, he has three book chapters to his credit. He is a reviewer for many reputed international journals including Int. J. Num. Methods for Heat \& Fluid Flow and Int. J. Heat \& Mass Transfer.

\section{Introduction}

It is necessary to have deep understanding about the mechanism of fluid flow through a pipe, as it is extensively used in advanced engineering systems, industry and our day-to-day life. In human body the blood is flowing continuously through the arteries and veins. All the modern biomedical instruments like artificial hearts and dialysis systems work on the basis of fluid flow through pipes. In refrigeration and air conditioning application, refrigerants are flowing through pipes. On a broader scale, flow through pipes play an important role in the design and analysis of engineering systems. Burgers' equation which can be used to study the flow through pipes is an important model. The Navier-Stokes equation is considered to be a cornerstone in fluid mechanics which, when expressed in its originality is a set of unsteady, nonlinear, second-order partial differential equations. Burgers' equation whose exact solution is well-known, can be considered as a simplified form of the one-dimensional Navier-Stokes equation. Burgers' model is suitable for the analysis in gas dynamics, shock wave theory, cosmology and traffic flow 
(Collier et al., 2013; Dhawan et al., 2012; Kardar et al., 1986; Molchanov et al., 1997; Molchanov et al., 1995; Shandarin and Zeldovich, 1989; Vergassola et al., 1994). The application of this model in various similar important fields, always require the solution of basic Burgers' equation.

In 1915, Harry Bateman (1882-1946), an English mathematician, introduced the following equation in his paper along with its corresponding initial and boundary conditions given by equations (1) to (3).

$$
\begin{aligned}
& \frac{\partial u}{\partial t}+u \frac{\partial u}{\partial x}=v \frac{\partial^{2} u}{\partial x^{2}}, \quad 0<x<L, 0<t<\tau \\
& u(x, 0)=\psi(x), \quad 0<x<L \\
& u(0, t)=\zeta_{1}(t), \quad u(L, t)=\zeta_{2}(t), \quad 0<t<\tau
\end{aligned}
$$

where $u, x, t$ and $v$ are the velocity, spatial coordinate, time and kinematic viscosity, respectively. The $\psi, \zeta_{1}$ and $\zeta_{2}$ are prescribed functions of variables depending upon the specific conditions for the problem to be solved. Later in 1948, Johannes Martinus Burgers (1895-1981) (Burgers, 1939, 1948; Nieuwstadt and Steketee, 1995), a Dutch physicist, explained the mathematical modelling of turbulence with the help of equation (1). In order to honour the contributions of Burgers, this equation is well-known as the 'Burgers' equation'. The simultaneous presence of nonlinear convective term $\left(u \frac{\partial u}{\partial x}\right)$ and diffusive term $\left(v \frac{\partial^{2} u}{\partial x^{2}}\right)$ add an additional feature to the Burgers' equation. When $v$ approaches zero, equation (1) become inviscid Burgers' equation which is a model for nonlinear wave propagation. When $u$ approach zero, equation (1) become the heat equation.

Julian David Cole (1925-1999) (Cole, 1951) and E. Hopf (1902-1983) (Hopf, 1950) independently introduced a transformation to convert Burgers' equation into a linear heat equation and solved exactly for an arbitrary initial condition. Hence, the transformation is famously known as Hopf-Cole transformation given by equation (4).

$$
u(x, t)=-2 v \frac{\theta x}{\theta},
$$

where, $\theta$ satisfy the heat equation

$$
\frac{\partial \theta}{\partial t}=v \frac{\partial^{2} \theta}{\partial x^{2}}
$$

Benton and Platman (1972) have been given 35 distinct analytical solutions of Burgers' equation with different initial conditions. Rodin (1970) studied some approximate and exact solution of boundary value problem for Burgers' equation with the help of Hopf-Cole transformation. Kutluay et al. (1999) used Hopf-Cole transformation to convert Burgers' equation to a heat equation. The transformed heat equation with the insulated boundary conditions was solved by explicit and exact-explicit finite difference method. Bakodah (2011) used MOL semi-discretisation approach to transform partial differential equations into a system of first order linear ordinary differential equations. The main contribution by Bakodah was the use of 7-point formula in MOL for solving Burgers' equation with arbitrary initial conditions. MOL is used by various authors to 
solve the system of ODE and PDE (Kurtz et al., 1977; Oymak and Selcuk, 1996; Rothe, 1930; Shampine, 1994; Shu, 1988; Spiteri and Ruuth, 2002). Burns et al. (1998) have been considered Burgers' equation with zero-Neumann boundary conditions to show that, for moderate values of the viscosity, numerical solution approach non-constant shock type stationary solution. Based on Hopf-Cole linearisation, Brander and Hedenfalk (1998) solved Burgers' equation in one space dimension for an arbitrary incident pulse of finite length. Restrictive Pade approximation classical implicit finite difference method was implemented by Gulsu (2006) whose accuracy was demonstrated by the two test problem.

The structure of this paper includes Section 2 which gives details of proposed difference scheme for Burgers' equation. This section is split into two parts; an implicit box scheme with Hopf-Cole transformation and the proposed implicit scheme without Hopf-Cole transformation. To obtain the finite difference solution of Burgers' equation, the differential equation at each node is replaced by a difference equation. After considering boundary conditions in the difference equations, the resulting algebraic system of equations is solved. In Section 3, the accuracy of the proposed scheme is verified by performing several numerical experiments and calculating $L_{2}$ and $L_{\infty}$ errors. Section 4 contains results and discussion of numerical experiments performed with test examples. Conclusions arrived based on the presented work are elaborated in Section 5.

\section{Difference scheme}

The solution domain is discretised with uniform meshes. The space interval $[0,1]$ is divided into $N$ equal subintervals. The time interval $[0, \tau]$ is divided into $M$ equal sub-intervals. Assuming $\Delta x=1 / N$ as the mesh width in space and $x_{i}$ is set as $x_{i}=i \Delta x$ for $i=0,1, \ldots, N$. Assuming $\Delta t=\tau / M$ as the mesh width in time and $t^{n}$ is set as $t^{n}=n \Delta t$ for $n=0,1, \ldots, M$.

\subsection{Implicit box scheme with Hopf-Cole transformation}

By using Hop-Cole transformation Burgers' equation is converted to heat equation (Kadalbajoo and Awasthi, 2006) which is then solved with the help of modified Keller box method (Bonkile et al., 2015). In Keller box scheme (Keller, 1970), second and higher derivatives of parabolic partial differential equation are replaced by first derivatives through the introduction of additional variables which result in a system of first-order equations. Equation (5) is written as a system of two first-order equations:

$$
\begin{aligned}
& \frac{\partial \theta}{\partial t}=v \frac{\partial T}{\partial x} \\
& \frac{\partial \theta}{\partial x}=T
\end{aligned}
$$

We use central differences about $\left(i-\frac{1}{2}, n+\frac{1}{2}\right)$, making use of four points at the corners of a 'box' [Figure 1(a)]. The resulting difference equations for equations (6) and (7) are 


$$
\begin{aligned}
& \frac{\theta_{i}^{n+1}-\theta_{i-1}^{n+1}}{\Delta x}=T_{i-\frac{1}{2}}^{n+1} \\
& \frac{\theta_{i-\frac{1}{2}}^{n+1}-\theta_{i-\frac{1}{2}}^{n}}{\Delta t}=v\left(\frac{T_{i}^{n+\frac{1}{2}}-T_{i-1}^{n+\frac{1}{2}}}{\Delta x}\right)
\end{aligned}
$$

The discretised terms containing subscript or superscript $\frac{1}{2}$ in equations (8) and (9) are defined as averages, for example,

$$
\begin{aligned}
& \theta_{i-\frac{1}{2}}^{n+1}=\frac{\theta_{i}^{n+1}+\theta_{i-1}^{n+1}}{2} \\
& T_{i}^{n+\frac{1}{2}}=\frac{T_{i}^{n}+T_{i}^{n+1}}{2}
\end{aligned}
$$

Figure 1 (a) Grid for box scheme (b) Difference molecule for evaluation of $T_{i-\frac{1}{2}}^{n+1}$ (c) Difference molecule for equation (9) (see online version for colours)

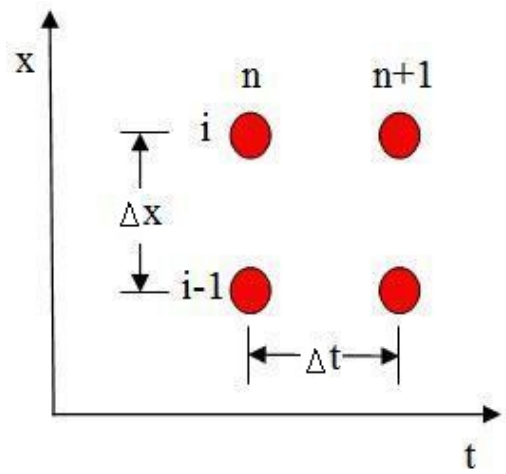

(a)

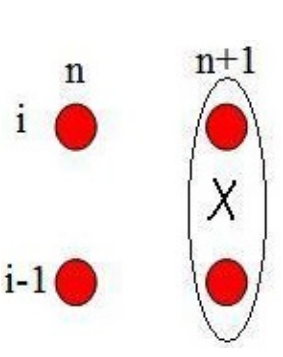

(b)

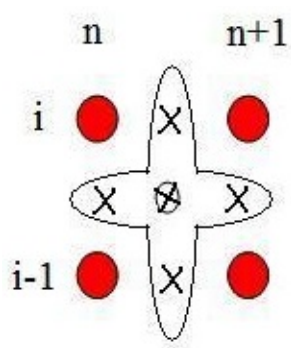

(c)

Averaged expressions (10) and (11) are substituted into equations (8) and (9). The resulting difference equations become

$$
\frac{\theta_{i}^{n+1}-\theta_{i-1}^{n+1}}{\Delta x}=\frac{T_{i}^{n+1}+T_{i-1}^{n+1}}{2}
$$




$$
\frac{\theta_{i}^{n+1}+\theta_{i-1}^{n+1}}{\Delta t}=v\left(\frac{T_{i}^{n}-T_{i-1}^{n}}{\Delta x}\right)+\frac{\theta_{i}^{n}+\theta_{i-1}^{n}}{\Delta t}+v\left(\frac{T_{i}^{n+1}-T_{i-1}^{n+1}}{\Delta x}\right)
$$

In equations (12) and (13) T's can be express in terms of $\theta$ 's. Substituting equation (12) into equation (13), $T_{i-1}^{n+1}$ is eliminated. Equation (12) is evaluated at time level $n$ to eliminate $T_{i-1}^{n}$. Accordingly

$$
\begin{aligned}
\frac{\theta_{i}^{n+1}+\theta_{i-1}^{n+1}}{\Delta t}= & v\left(\frac{T_{i}^{n}+T_{i}^{n+1}}{\Delta x}\right)+\frac{\theta_{i}^{n}+\theta_{i-1}^{n}}{\Delta t} \\
& -v\left(\frac{\theta_{i}^{n+1}-\theta_{i-1}^{n+1}}{(\Delta x)^{2}}-\frac{T_{i}^{n+1}}{\Delta x}+2 \frac{\theta_{i}^{n}-\theta_{i-1}^{n}}{(\Delta x)^{2}}-\frac{T_{i}^{n}}{\Delta x}\right)
\end{aligned}
$$

Equations (12) and (13) are rewritten with the $i$ index advanced by 1 .

$$
\begin{aligned}
& \frac{\theta_{i+1}^{n+1}-\theta_{i}^{n+1}}{\Delta x}=\frac{T_{i+1}^{n+1}+T_{i}^{n+1}}{2} \\
& \frac{\theta_{i+1}^{n+1}+\theta_{i}^{n+1}}{\Delta t}=v\left(\frac{T_{i+1}^{n}-T_{i}^{n}}{\Delta x}\right)+\frac{\theta_{i+1}^{n}+\theta_{i}^{n}}{\Delta t}+v\left(\frac{T_{i+1}^{n+1}-T_{i}^{n+1}}{\Delta x}\right)
\end{aligned}
$$

To eliminate $T_{i+1}^{n+1}$ and $T_{i+1}^{n}$, equation (15) is simply substituted into equation (16). The result is

$$
\frac{\theta_{i+1}^{n+1}+\theta_{i}^{n+1}}{\Delta t}=2 v\left(\frac{\left(\theta_{i+1}^{n}-\theta_{i}^{n}\right)}{(\Delta x)^{2}}-\frac{T_{i}^{n}}{\Delta x}\right)+\frac{\theta_{i+1}^{n}+\theta_{i}^{n}}{\Delta t}+2 v\left(\frac{\left(\theta_{i+1}^{n+1}-\theta_{i}^{n+1}\right)}{(\Delta x)^{2}}-\frac{T_{i}^{n+1}}{\Delta x}\right)
$$

Adding equations (14) and (17), we have

$$
\begin{aligned}
& \left(1-\frac{2 v \Delta t}{(\Delta x)^{2}}\right) \theta_{i-1}^{n+1}+\left(2+\frac{4 v \Delta t}{(\Delta x)^{2}}\right) \theta_{i}^{n+1}+\left(1-\frac{2 v \Delta t}{(\Delta x)^{2}}\right) \theta_{i+1}^{n+1} \\
& =\left(1+\frac{2 v \Delta t}{(\Delta x)^{2}}\right) \theta_{i-1}^{n}+\left(2-\frac{4 v \Delta t}{(\Delta x)^{2}}\right) \theta_{i}^{n}+\left(1+\frac{2 v \Delta t}{(\Delta x)^{2}}\right) \theta_{i+1}^{n}
\end{aligned}
$$

Equation (18) can be written in tridiagonal form as

$$
a_{i} \theta_{i-1}^{n+1}+b_{i} \theta_{i}^{n+1}+c_{i} \theta_{i+1}^{n+1}=d_{i}
$$

where,

$$
\begin{aligned}
& a_{i}=1-2 \frac{v \Delta t}{(\Delta x)^{2}}=\left(1-2 F_{0}\right) \\
& b_{i}=2+4 \frac{v \Delta t}{(\Delta x)^{2}}=2\left(1+2 F_{0}\right) \\
& c_{i}=1-2 \frac{v \Delta t}{(\Delta x)^{2}}=\left(1-2 F_{0}\right)
\end{aligned}
$$




$$
\begin{aligned}
d_{i} & =\left(1+2 \frac{v \Delta t}{(\Delta x)^{2}}\right) \theta_{i-1}^{n}+\left(2-4 \frac{v \Delta t}{(\Delta x)^{2}}\right) \theta_{i}^{n}+\left(1+2 \frac{v \Delta t}{(\Delta x)^{2}}\right) \theta_{i+1}^{n} \\
& =\left(1+2 F_{0}\right)\left(\theta_{i-1}^{n}+\theta_{i+1}^{n}\right)+2\left(1-2 F_{0}\right) \theta_{i}^{n}
\end{aligned}
$$

Here $F_{0}=\frac{v \Delta t}{(\Delta x)^{2}}$ is the Fourier number which is otherwise known as the dimensionless time. After assembling the entire system of equations and applying boundary conditions, the matrix form of the equation is $F \theta=d$. Where $F$ is a tridiagonal matrix of order $(N \times N)$ and $d$ and $\theta$ are column vectors.

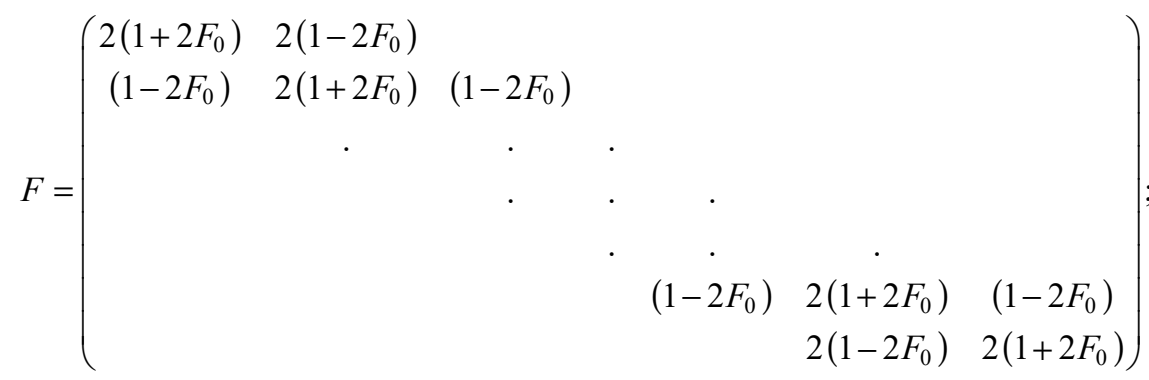

$$
\begin{aligned}
& \theta=\left(\begin{array}{c}
\theta_{1}^{n+1} \\
\theta_{2}^{n+1} \\
\cdot \\
\cdot \\
\cdot \\
\theta_{N-1}^{n+1} \\
\theta_{N}^{n+1}
\end{array}\right) ; \quad d=\left(\begin{array}{c}
\left(1+2 F_{0}\right)\left(\theta(0, t)+\theta_{2}^{n}\right)+2\left(1-2 F_{0}\right) \theta_{1}^{n} \\
\left(1+2 F_{0}\right)\left(\theta_{1}^{n}+\theta_{3}^{n}\right)+2\left(1-2 F_{0}\right) \theta_{2}^{n} \\
\cdot \\
\cdot \\
\cdot \\
\left(1+2 F_{0}\right)\left(\theta_{N-2}^{n}+\theta_{N}^{n}\right)+2\left(1-2 F_{0}\right) \theta_{N-1}^{n} \\
\left(1+2 F_{0}\right)\left(\theta_{N-1}^{n}+\theta(1, t)\right)+2\left(1-2 F_{0}\right) \theta_{N}^{n}
\end{array}\right)
\end{aligned}
$$

The approximate solution of Burgers' equation (1) in terms of the approximate solution of heat equation by using the Hopf-Cole transformation (4) is given by

$$
u_{i}^{n}=-v\left(\frac{\theta_{i+1}^{n}-\theta_{i-1}^{n}}{\Delta x \theta_{i}^{n}}\right)
$$

\subsection{Implicit scheme without Hopf-Cole transformation}

In this approach, an implicit scheme similar to box method is directly implemented to nonlinear Burgers' equation. In equation (1), let us substitute

$$
\frac{\partial u}{\partial x}=\Phi
$$

Equation (1) become

$$
\frac{\partial u}{\partial t}+u \Phi=v \frac{\partial \Phi}{\partial x}
$$


We use central differences about $\left(i-\frac{1}{2}, n+\frac{1}{2}\right)$. The resulting difference equations for equations (25) and (26) are

$$
\begin{aligned}
& \frac{u_{i}^{n+1}-u_{i-1}^{n+1}}{\Delta x}=\Phi_{i-\frac{1}{2}}^{n+1} \\
& \frac{u_{i-\frac{1}{2}}^{n+1}-u_{i-\frac{1}{2}}^{n}}{\Delta t}+u_{i-\frac{1}{2}}^{n} \Phi_{i-\frac{1}{2}}^{n+1}=v\left(\frac{\Phi_{i}^{n+\frac{1}{2}}-\Phi_{i-1}^{n+\frac{1}{2}}}{\Delta x}\right)
\end{aligned}
$$

The discretised terms containing subscript or superscript $\frac{1}{2}$ in equations (27) and (28) are defined as averages, for example,

$$
\begin{gathered}
\Phi_{i-\frac{1}{2}}^{n+1}=\frac{\Phi_{i}^{n+1}+\Phi_{i-1}^{n+1}}{2} \\
u_{i-\frac{1}{2}}^{n}=\frac{u_{i}^{n}+u_{i-1}^{n}}{2}
\end{gathered}
$$

After substituting average equations (29) and (30) into equations (25) and (26), the resulting difference equations are

$$
\begin{aligned}
\frac{u_{i}^{n+1}-u_{i-1}^{n+1}}{\Delta x}= & \frac{\Phi_{i}^{n+1}+\Phi_{i-1}^{n+1}}{2} \\
\frac{u_{i}^{n+1}+u_{i-1}^{n+1}}{\Delta t}= & v\left(\frac{\Phi_{i}^{n}+\Phi_{i}^{n+1}-\Phi_{i-1}^{n}-\Phi_{i-1}^{n+1}}{\Delta x}\right)+\frac{u_{i}^{n}+u_{i-1}^{n}}{\Delta t} \\
& -\left(u_{i}^{n}+u_{i-1}^{n}\right)\left(\frac{\Phi_{i}^{n+1}+\Phi_{i-1}^{n+1}}{2}\right)
\end{aligned}
$$

$\Phi_{i-1}^{n+1}$ is eliminated from equation (32) by simply substituting equation (31). Similarly $\Phi_{i-1}^{n}$ is eliminated by evaluating equation (31) at time level $n$. Accordingly

$$
\begin{aligned}
\frac{u_{i}^{n+1}+u_{i-1}^{n+1}}{\Delta t}= & v\left(\frac{2 \Phi_{i}^{n}}{\Delta x}+\frac{2 \Phi_{i}^{n+1}}{\Delta x}-\frac{2\left(u_{i}^{n}-u_{i-1}^{n}\right)}{\Delta x^{2}}-\frac{2\left(u_{i}^{n+1}-u_{i-1}^{n+1}\right)}{\Delta x^{2}}\right) \\
& +\frac{u_{i}^{n}+u_{i-1}^{n}}{\Delta t}-\left(\frac{\left(u_{i}^{n}+u_{i-1}^{n}\right)\left(u_{i}^{n+1}-u_{i-1}^{n+1}\right)}{\Delta x}\right)
\end{aligned}
$$

To eliminate $\Phi_{i+1}^{n+1}$ and $\Phi_{i+1}^{n}$, equations (31) and (32) are first rewritten with $i$ index advanced by 1 .

$$
\frac{u_{i+1}^{n+1}-u_{i}^{n+1}}{\Delta x}=\frac{\Phi_{i+1}^{n+1}+\Phi_{i}^{n+1}}{2}
$$




$$
\begin{aligned}
\frac{u_{i+1}^{n+1}+u_{i}^{n+1}}{\Delta t}= & v\left(\frac{\Phi_{i+1}^{n}-\Phi_{i}^{n}+\Phi_{i+1}^{n+1}-\Phi_{i}^{n+1}}{\Delta x}\right)+\frac{u_{i+1}^{n}+u_{i}^{n}}{\Delta t} \\
& -\frac{\left(u_{i+1}^{n}+u_{i}^{n}\right)\left(\Phi_{i+1}^{n+1}+\Phi_{i}^{n+1}\right)}{2}
\end{aligned}
$$

Equation (34) is substituted in equation (35), to eliminated $\Phi_{i+1}^{n+1}$ and $\Phi_{i+1}^{n}$

$$
\begin{aligned}
u_{i+1}^{n+1}+u_{i}^{n+1}= & v\left(\frac{2\left(u_{i+1}^{n}-u_{i}^{n}\right)}{\Delta x^{2}}-\frac{2 \Phi_{i}^{n}}{\Delta x}+\frac{2\left(u_{i+1}^{n+1}-u_{i}^{n+1}\right)}{\Delta x^{2}}-\frac{2 \Phi_{i}^{n+1}}{\Delta x}\right) \\
& +\frac{u_{i+1}^{n}+u_{i}^{n}}{\Delta t}-\frac{\left(u_{i+1}^{n}+u_{i}^{n}\right)\left(u_{i+1}^{n+1}-u_{i}^{n+1}\right)}{\Delta x}
\end{aligned}
$$

Adding equations (33) and (36), the rearranged final expression is

$$
\begin{aligned}
& {\left[1-\frac{2 v \Delta t}{(\Delta x)^{2}}-\frac{\Delta t}{\Delta x}\left(u_{i}^{n}+u_{i-1}^{n}\right)\right] u_{i-1}^{n+1}+\left[2+2 \frac{2 v \Delta t}{(\Delta x)^{2}}+\frac{\Delta t}{\Delta x}\left(u_{i-1}^{n}+u_{i-1}^{n}\right)\right] u_{i}^{n+1}} \\
& +\left[1-\frac{2 v \Delta t}{(\Delta x)^{2}}+\frac{\Delta t}{\Delta x}\left(u_{i+1}^{n}+u_{i}^{n}\right)\right] u_{i+1}^{n+1}=\left[1+\frac{2 v \Delta t}{(\Delta x)^{2}}\right] u_{i-1}^{n}+\left[2-2 \frac{2 v \Delta t}{(\Delta x)^{2}}\right] u_{i}^{n} \\
& +\left[1+\frac{2 v \Delta t}{(\Delta x)^{2}}\right] u_{i+1}^{n}
\end{aligned}
$$

Equation (37) can be written in tridiagonal form as

$$
A_{i} u_{i-1}^{n+1}+B_{i} u_{i}^{n+1}+C_{i} u_{i+1}^{n+1}=D_{i}
$$

where,

$$
\begin{aligned}
A_{i} & =1-2 \frac{v \Delta t}{(\Delta x)^{2}}-\frac{\Delta t}{\Delta x}\left(u_{i}^{n}+u_{i-1}^{n}\right) \\
& =1-2 F_{0}-\frac{\Delta t}{\Delta x}\left(u_{i}^{n}+u_{i-1}^{n}\right) \\
B_{i} & =2+4 \frac{v \Delta t}{(\Delta x)^{2}}+\frac{\Delta t}{\Delta x}\left(u_{i-1}^{n}+u_{i-1}^{n}\right) \\
& =2+4 F_{0}+\frac{\Delta t}{\Delta x}\left(u_{i-1}^{n}+u_{i-1}^{n}\right) \\
C_{i} & =1-2 \frac{v \Delta t}{(\Delta x)^{2}}+\frac{\Delta t}{\Delta x}\left(u_{i+1}^{n}+u_{i}^{n}\right) \\
& =1-2 F_{0}+\frac{\Delta t}{\Delta x}\left(u_{i+1}^{n}+u_{i}^{n}\right) \\
D_{i} & =\left[1+2 \frac{v \Delta \tau}{(\Delta x)^{2}}\right] u_{i-1}^{n}+\left[2-4 \frac{v \Delta t}{(\Delta x)^{2}}\right] u_{i}^{n}+\left[1+2 \frac{v \Delta t}{(\Delta x)^{2}}\right] u_{i+1}^{n} \\
& =\left(1+2 F_{0}\right) u_{i-1}^{n}+\left(2-4 F_{0}\right) u_{i}^{n}+\left(1+2 F_{0}\right) u_{i+1}^{n}
\end{aligned}
$$


Figure 2 Flow chart for the algorithm with Hopf-Cole transformation (see online version for colours)

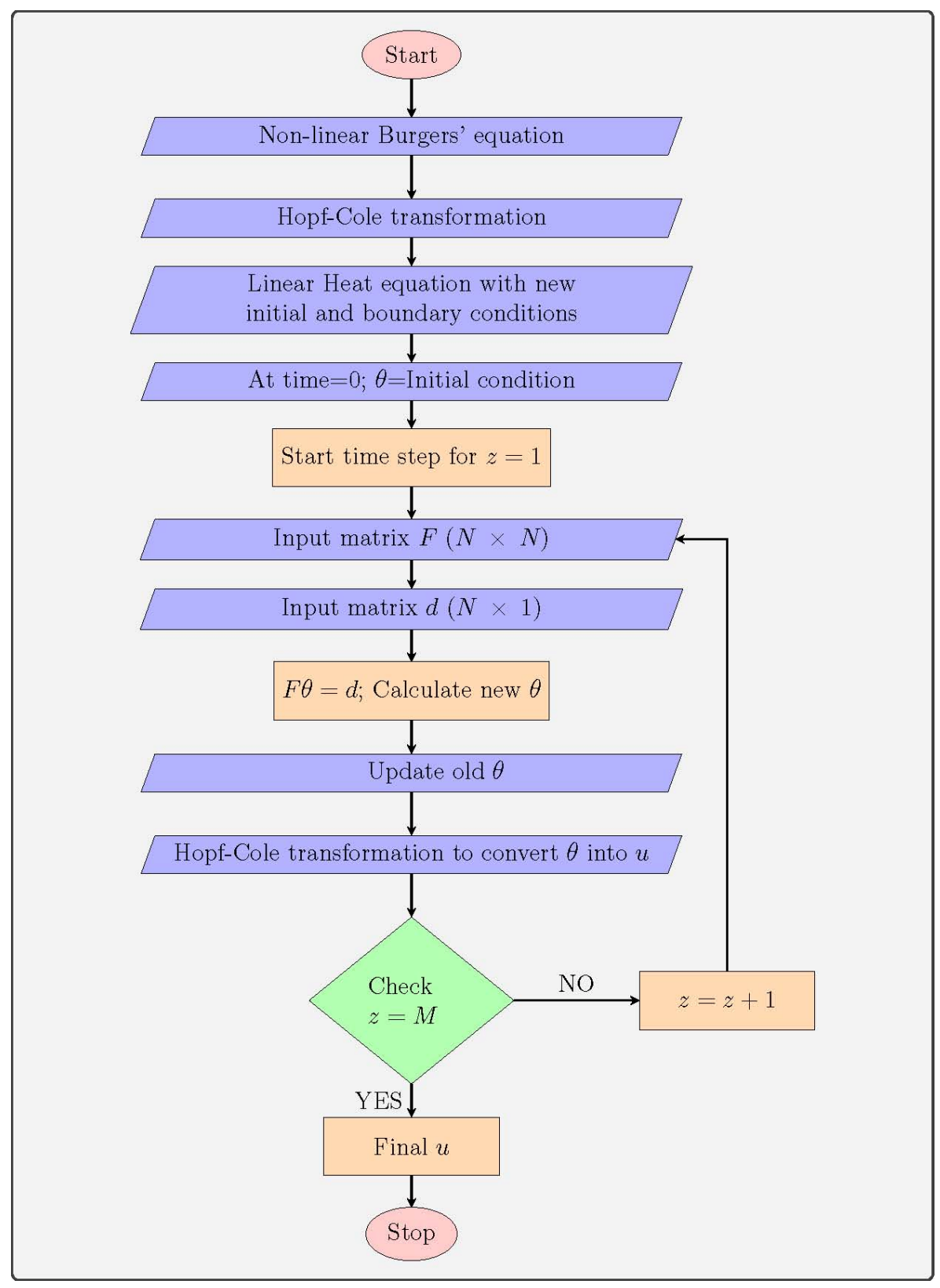


Figure 3 Flow chart for the new implicit scheme (see online version for colours)

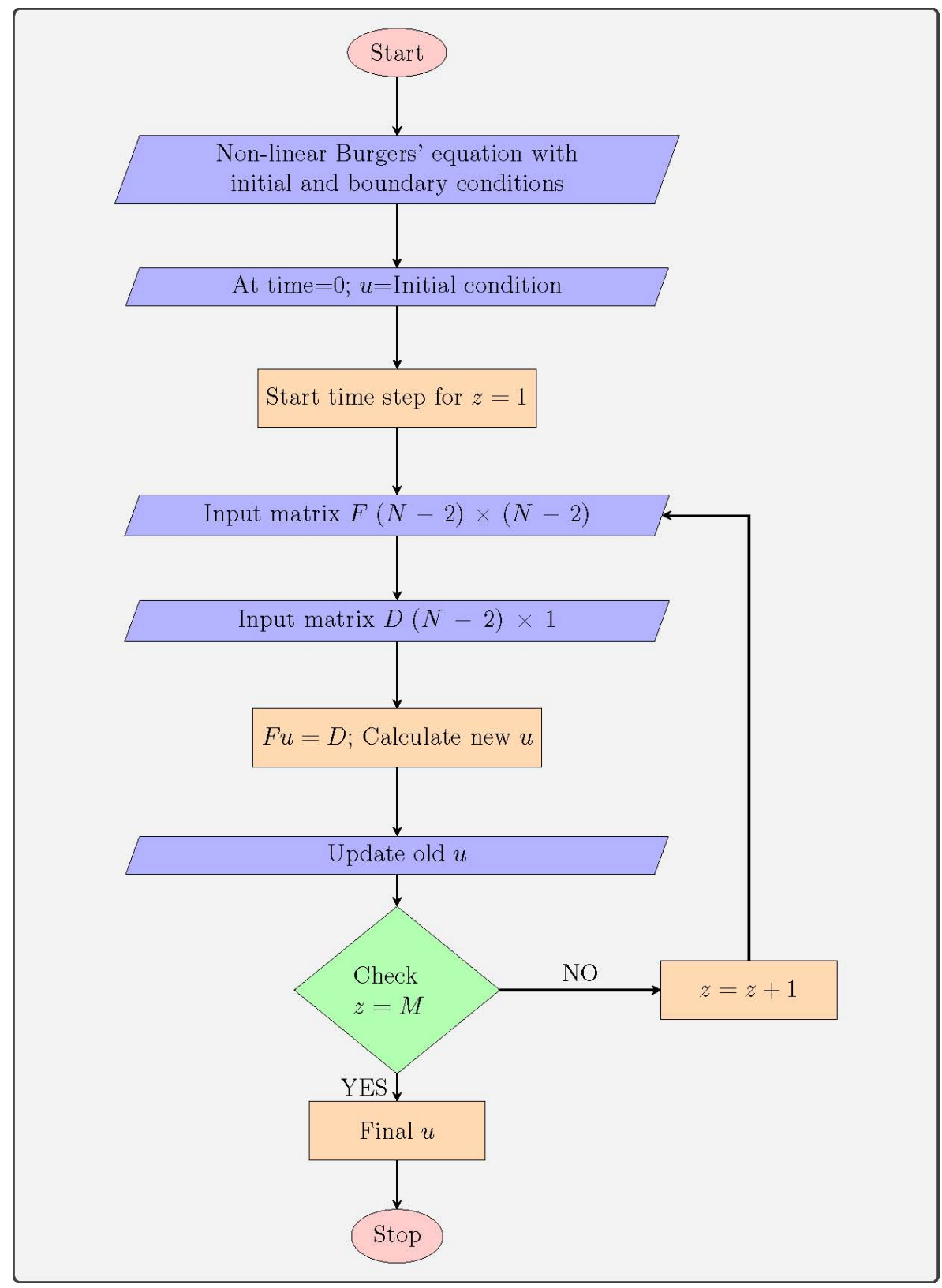


After assembling the entire system of equations and applying boundary conditions, the general matrix form is $F u=D$. Where $F$ is a tridiagonal matrix of order $(N-2) \times(\mathrm{N}-2)$, $D$ and $u$ are the vectors.

$$
\begin{aligned}
& F=\left(\begin{array}{ccccccc}
B_{2} & C_{2} & & & & & \\
A_{3} & B_{3} & C_{3} & & & & \\
& \cdot & \cdot & \cdot & & & \\
& & \cdot & \cdot & \cdot & & \\
& & & \cdot & \cdot & \cdot & \\
& & & & A_{N-2} & B_{N-2} & C_{N-2} \\
& & & & & A_{N-1} & B_{N-1}
\end{array}\right) ; \quad u=\left(\begin{array}{c}
u_{2}^{n+1} \\
u_{3}^{n+1} \\
\cdot \\
\cdot \\
\cdot \\
u_{N-2}^{n+1} \\
u_{N-1}^{n+1}
\end{array}\right) ; \\
& D=\left(\begin{array}{c}
\left(1+2 F_{0}\right) u_{1}^{n}+\left(2-4 F_{0}\right) u_{2}^{n}+\left(1+2 F_{0}\right) u_{3}^{n} \\
\left(1+2 F_{0}\right) u_{2}^{n}+\left(2-4 F_{0}\right) u_{3}^{n}+\left(1+2 F_{0}\right) u_{4}^{n} \\
\cdot \\
\cdot \\
\cdot \\
\left(1+2 F_{0}\right) u_{N-3}^{n}+\left(2-4 F_{0}\right) u_{N-2}^{n}+\left(1+2 F_{0}\right) u_{N-1}^{n} \\
\left(1+2 F_{0}\right) u_{N-2}^{n}+\left(2-4 F_{0}\right) u_{N-1}^{n}+\left(1+2 F_{0}\right) u_{N}^{n}
\end{array}\right)
\end{aligned}
$$

\section{Numerical experiments}

After presenting the proposed scheme, here the simulation study of two test examples is undertaken to validate the theoretical results obtained by the new scheme without Hopf-Cole transformation. Due to its transient nature, Burgers' equation is difficult for carrying out the analyses. The boundary conditions have sufficient smoothness for maintaining the order of accuracy of the proposed scheme. Matlab software is used to programme and generate the numerical solutions of the boundary value problems. A small time increment $(\Delta t=0.00001,0.0001,0.01)$ is chosen to insure high accuracy. Also, $L_{2}$ and $L_{\infty}$ errors are evaluated to measure the accuracy and the efficiency of the proposed method. $L_{2}$ and $L_{\infty}$ errors are defined as

$$
L_{2}=\left(\sum_{i=1}^{N}\left[u_{i}^{\text {exact }}-u_{i}^{+}\right]^{2}\right)^{\frac{1}{2}}, \quad L_{\infty}=\max _{1 \leq i \leq N}\left|u_{i}^{\text {exact }}-u_{i}^{+}\right|
$$

where $u_{i}^{+}$represent the numerical solution at node $i$.

Example 1: Burgers' equation (1) with initial condition and homogeneous boundary conditions

$$
\begin{aligned}
& u(x, 0)=\sin (\pi x), \quad 0<x<1, \\
& u(0, t)=u(1, t)=0, \quad 0 \leq t \leq \tau
\end{aligned}
$$


By using Hopf-Cole transformation (4), equation (1) is transformed to the linear heat equation (5) with initial condition (44) and boundary conditions (45)

$$
\begin{aligned}
& \theta(x, 0)=\exp \left(-\frac{1}{2 \pi \nu}[1-\cos (\pi x)]\right), \quad 0<x<1 \\
& \theta_{x}(0, t)=\theta_{x}(1, t)=0, \quad 0 \leq t \leq \tau
\end{aligned}
$$

Example 2: Burgers' equation (1) with the following initial condition and boundary conditions

$$
\begin{array}{ll}
u(x, 0)=4 x(1-x), & 0<x<1, \\
u(0, t)=0=u(1, t), & 0 \leq t \leq \tau .
\end{array}
$$

By using Hopf-Cole transformation (4), equation (1) is transformed to linear heat equation (5) with initial condition and boundary conditions

$$
\begin{aligned}
& \theta(x, 0)=\exp \left(-\frac{1}{2 v}\left[2 x^{2}-\frac{4 x^{3}}{3}\right]\right), \quad 0<x<1 \\
& \theta_{x}(0, t)=\theta_{x}(1, t)=0, \quad 0 \leq t \leq \tau .
\end{aligned}
$$

Exact solution was elaborated by Kadalbajoo and Awasthi (2006) for both Examples 1 and 2.

\section{Results and discussion}

Burgers' equation being a nonlinear PDE represents various physical problems arising in engineering which are inherently difficult to solve. This section contains results and discussion of numerical experiments performed on test examples. $L_{2}$ and $L_{\infty}$ error are calculated at $N=10,20,40,80$ for both the schemes. Tables 1 to 4 display comparison of $L_{2}$ and $L_{\infty}$ error calculated for scheme with and without Hopf-Cole transformation considering test example 1 . For various values of $v$ and $\Delta t$, comparison of $L_{2}$ and $L_{\infty}$ error calculated for scheme with and without Hopf-Cole transformation considering test example 2 is shown in Tables 5 to 8 . Four different values of kinematic viscosity $(v=0.005,0.01,0.1,1)$ is considered in increasing order. In most of the cases it is observed that, $L_{2}$ and $L_{\infty}$ error of numerical solution obtained without Hopf-Cole transformation is less. $L_{\infty}$ error is significantly reduced for the proposed scheme without Hopf-Cole transformation. It is observed that, the proposed implicit scheme without Hopf-Cole transformation performs well as compared to modified box scheme coupled with Hopf-Cole transformation. In Figure 4 and 5, graphs are plotted for error vs. $N$. Blue and green lines represent $L_{2}$ error without and with Hopf-Cole transformation respectively. Whereas red and yellow lines represent $L_{\infty}$ error without and with Hopf-Cole transformation respectively. It is clear from these graphs that, at $N=40$ both $L_{2}$ and $L_{\infty}$ error decrease suddenly. It is found that the computed results show better agreement with the exact solution as the mesh size is refined. 
Table 1 Comparison of the $L_{2}$ and $L_{\infty}$ error of ex. 1 for $v=0.005, \tau=7$ and $\Delta t=0.01$

\begin{tabular}{|c|c|c|c|c|}
\hline & \multicolumn{2}{|c|}{$N=10$} & \multicolumn{2}{|c|}{$N=20$} \\
\hline & With Hopf-Cole & Without Hopf-Cole & With Hopf-Cole & Without Hopf-Cole \\
\hline$L_{2}$ & 0.03013 & $6.30065 \times 10^{-4}$ & 0.00599 & $1.86747 \times 10^{-4}$ \\
\hline \multirow[t]{3}{*}{$L_{\infty}$} & 0.24240 & 0.00540 & 0.11420 & 0.00341 \\
\hline & \multicolumn{2}{|c|}{$N=40$} & \multicolumn{2}{|c|}{$N=80$} \\
\hline & With Hopf-Cole & Without Hopf-Cole & With Hopf-Cole & Without Hopf-Cole \\
\hline$L_{2}$ & $3.23644 \times 10^{-4}$ & $3.46470 \times 10^{-5}$ & $6.32711 \times 10^{-5}$ & $1.31974 \times 10^{-5}$ \\
\hline$L_{\infty}$ & 0.00532 & $7.04929 \times 10^{-4}$ & 0.00142 & $2.73112 \times 10^{-4}$ \\
\hline
\end{tabular}

Table 2 Comparison of the $L_{2}$ and $L_{\infty}$ error of ex. 1 for $v=0.01, \tau=5$ and $\Delta t=0.01$

\begin{tabular}{|c|c|c|c|c|}
\hline & \multicolumn{2}{|c|}{$N=10$} & \multicolumn{2}{|c|}{$N=20$} \\
\hline & With Hopf-Cole & Without Hopf-Cole & With Hopf-Cole & Without Hopf-Cole \\
\hline$L_{2}$ & 0.04832 & $8.20495 \times 10^{-4}$ & $8.88056 \times 10^{-4}$ & $1.56212 \times 10^{-4}$ \\
\hline \multirow[t]{3}{*}{$L_{\infty}$} & 0.47831 & 0.00744 & 0.01004 & 0.00236 \\
\hline & \multicolumn{2}{|c|}{$N=40$} & \multicolumn{2}{|c|}{$N=80$} \\
\hline & With Hopf-Cole & Without Hopf-Cole & With Hopf-Cole & Without Hopf-Cole \\
\hline$\overline{L_{2}}$ & $1.70411 \times 10^{-4}$ & $3.81897 \times 10^{-5}$ & $4.53729 \times 10^{-5}$ & $2.42870 \times 10^{-5}$ \\
\hline$L_{\infty}$ & 0.00264 & $5.12893 \times 10^{-4}$ & $9.35877 \times 10^{-4}$ & $4.34366 \times 10^{-4}$ \\
\hline
\end{tabular}

Table 3 Comparison of the $L_{2}$ and $L_{\infty}$ error of ex. 1 for $\nu=0.1, \tau=1$ and $\Delta t=0.0001$

\begin{tabular}{ccccccc}
\hline & \multicolumn{3}{c}{$N=10$} & & \multicolumn{2}{c}{$N=20$} \\
\cline { 2 - 3 } \cline { 5 - 6 } & With Hopf-Cole & Without Hopf-Cole & & With Hopf-Cole & Without Hopf-Cole \\
\hline$L_{2}$ & 0.00223 & 0.00135 & & $3.91918 \times 10^{-4}$ & $2.38391 \times 10^{-4}$ \\
$L_{\infty}$ & 0.01000 & 0.00874 & & 0.00250 & 0.00218 \\
\cline { 2 - 3 } \cline { 6 - 7 } \cline { 5 - 7 } & \multicolumn{3}{c}{$N=40$} & & \multicolumn{2}{c}{$N=80$} \\
\hline$L_{2}$ & $6.65393 \times 10^{-5}$ & $4.04704 \times 10^{-5}$ & & $9.84522 \times 10^{-6}$ & $6.01207 \times 10^{-6}$ \\
$L_{\infty}$ & $6.04666 \times 10^{-4}$ & $5.36008 \times 10^{-4}$ & & $1.29917 \times 10^{-4}$ & $1.16958 \times 10^{-4}$ \\
\hline
\end{tabular}

Table 4 Comparison of the $L_{2}$ and $L_{\infty}$ error of ex. 1 for $v=1, \tau=0.1$ and $\Delta t=0.0001$

\begin{tabular}{ccccccc}
\hline & \multicolumn{3}{c}{$N=10$} & & \multicolumn{2}{c}{$N=20$} \\
\cline { 2 - 3 } \cline { 5 - 6 } & With Hopf-Cole & Without Hopf-Cole & & With Hopf-Cole & Without Hopf-Cole \\
\hline$L_{2}$ & 0.00261 & 0.00128 & & $4.21894 \times 10-4$ & $1.82668 \times 10-4$ \\
$L_{\infty}$ & 0.01170 & 0.00580 & & 0.00266 & 0.00117 \\
\cline { 2 - 3 } \cline { 6 - 7 } \cline { 5 - 6 } & \multicolumn{2}{c}{$N=40$} & & \multicolumn{2}{c}{$N=80$} \\
\hline$L_{2}$ & $4.40145 \times 10-5$ & $3.69330 \times 10-6$ & & $1.39859 \times 10-5$ & $2.13554 \times 10-5$ \\
$L_{\infty}$ & $3.94900 \times 10-4$ & $4.10527 \times 10-5$ & & $1.78162 \times 10-4$ & $2.70405 \times 10-4$ \\
\hline
\end{tabular}


Table 5 Comparison of the $L_{2}$ and $L_{\infty}$ error of ex. 2 for $v=0.005, \tau=7$ and $\Delta t=0.01$

\begin{tabular}{ccccccc}
\hline & \multicolumn{2}{c}{$N=10$} & & \multicolumn{2}{c}{$N=20$} \\
\cline { 2 - 3 } \cline { 5 - 6 } & With Hopf-Cole & Without Hopf-Cole & & With Hopf-Cole & Without Hopf-Cole \\
\hline$L_{2}$ & 0.02516 & $6.26644 \times 10^{-4}$ & & 0.01636 & $1.90023 \times 10^{-4}$ \\
$L_{\infty}$ & 0.16941 & 0.00534 & & 0.28872 & 0.00351 \\
\cline { 2 - 3 } \cline { 6 - 7 } \cline { 5 - 7 } & \multicolumn{2}{c}{$N=40$} & & \multicolumn{3}{c}{$N=80$} \\
\hline$L_{2}$ & $3.43914 \times 10^{-4}$ & $3.46009 \times 10^{-5}$ & & $6.50026 \times 10^{-5}$ & $1.27168 \times 10^{-5}$ \\
$L_{\infty}$ & 0.00658 & $7.33178 \times 10^{-4}$ & & 0.00148 & $2.65749 \times 10^{-4}$ \\
\hline
\end{tabular}

Table 6 Comparison of the $L_{2}$ and $L_{\infty}$ error of ex. 2 for $v=0.01, \tau=5$ and $\Delta t=0.01$

\begin{tabular}{|c|c|c|c|c|}
\hline & \multicolumn{2}{|c|}{$N=10$} & \multicolumn{2}{|c|}{$N=20$} \\
\hline & With Hopf-Cole & Without Hopf-Cole & With Hopf-Cole & Without Hopf-Cole \\
\hline$L_{2}$ & 0.07687 & $8.40126 \times 10^{-4}$ & $9.54599 \times 10^{-4}$ & $1.58959 \times 10^{-4}$ \\
\hline \multirow[t]{3}{*}{$L_{\infty}$} & 0.76142 & 0.00774 & 0.01337 & 0.00247 \\
\hline & \multicolumn{2}{|c|}{$N=40$} & \multicolumn{2}{|c|}{$N=80$} \\
\hline & With Hopf-Cole & Without Hopf-Cole & With Hopf-Cole & Without Hopf-Cole \\
\hline$L_{2}$ & $1.76817 \times 10^{-4}$ & $3.67044 \times 10^{-5}$ & $4.67206 \times 10^{-5}$ & $2.32107 \times 10^{-5}$ \\
\hline$L_{\infty}$ & 0.00283 & $4.91576 \times 10^{-4}$ & $9.66020 \times 10^{-4}$ & $4.18431 \times 10^{-4}$ \\
\hline
\end{tabular}

Table 7 Comparison of the $L_{2}$ and $L_{\infty}$ error of ex. 2 for $v=0.1, \tau=1$ and $\Delta t=0.0001$

\begin{tabular}{ccccccc}
\hline & \multicolumn{2}{c}{$N=10$} & & \multicolumn{2}{c}{$N=20$} \\
\cline { 2 - 3 } \cline { 5 - 6 } & With Hopf-Cole & Without Hopf-Cole & & With Hopf-Cole & Without Hopf-Cole \\
\hline$L_{2}$ & 0.00228 & 0.00140 & & $4.00813 \times 10^{-4}$ & $2.47679 \times 10^{-4}$ \\
$L_{\infty}$ & 0.01034 & 0.00910 & & 0.00256 & 0.00227 \\
\cline { 2 - 3 } \cline { 6 - 7 } \cline { 5 - 7 } & \multicolumn{2}{c}{$N=40$} & & \multicolumn{2}{c}{$N=80$} \\
\hline$L_{2}$ & $6.80527 \times 10^{-5}$ & $4.20617 \times 10^{-5}$ & & $1.00726 \times 10^{-5}$ & $6.26291 \times 10^{-6}$ \\
$L_{\infty}$ & $6.20440 \times 10^{-4}$ & $5.59172 \times 10^{-4}$ & & $1.33655 \times 10^{-4}$ & $1.22276 \times 10^{-4}$ \\
\hline
\end{tabular}

Table 8 Comparison of the $L_{2}$ and $L_{\infty}$ error of ex. 2 for $v=1, \tau=0.1$ and $\Delta t=0.0001$

\begin{tabular}{ccccccc}
\hline & \multicolumn{3}{c}{$N=10$} & & \multicolumn{2}{c}{$N=20$} \\
\cline { 2 - 3 } \cline { 5 - 6 } & With Hopf-Cole & Without Hopf-Cole & & With Hopf-Cole & Without Hopf-Cole \\
\hline$L_{2}$ & 0.00269 & 0.00132 & & $4.35078 \times 10^{-4}$ & $1.88513 \times 10^{-4}$ \\
$L_{\infty}$ & 0.01206 & 0.00600 & & 0.00275 & 0.00121 \\
\cline { 2 - 3 } \cline { 6 - 7 } \cline { 5 - 7 } & \multicolumn{3}{c}{$N=40$} & & \multicolumn{2}{c}{$N=80$} \\
\hline$L_{2}$ & With Hopf-Cole & Without Hopf-Cole & & With Hopf-Cole & Without Hopf-Cole \\
$L_{\infty}$ & $4.52541 \times 10^{-5}$ & $3.74359 \times 10^{-6}$ & & $1.45550 \times 10^{-5}$ & $2.21445 \times 10^{-5}$ \\
\hline
\end{tabular}


Figure $4 L_{2}$ and $L_{\infty}$ error of ex. 1 at (a) $\tau=0.1$ for $v=1$ and $\Delta t=0.0001$ (b) $\tau=5$ for $v=0.01$ and $\Delta t=0.01$ (c) $\tau=7$ for $v=0.005$ and $\Delta t=0.01$ (d) $\tau=1$ for $v=0.1$ and $\Delta t=0.0001$ (see online version for colours)

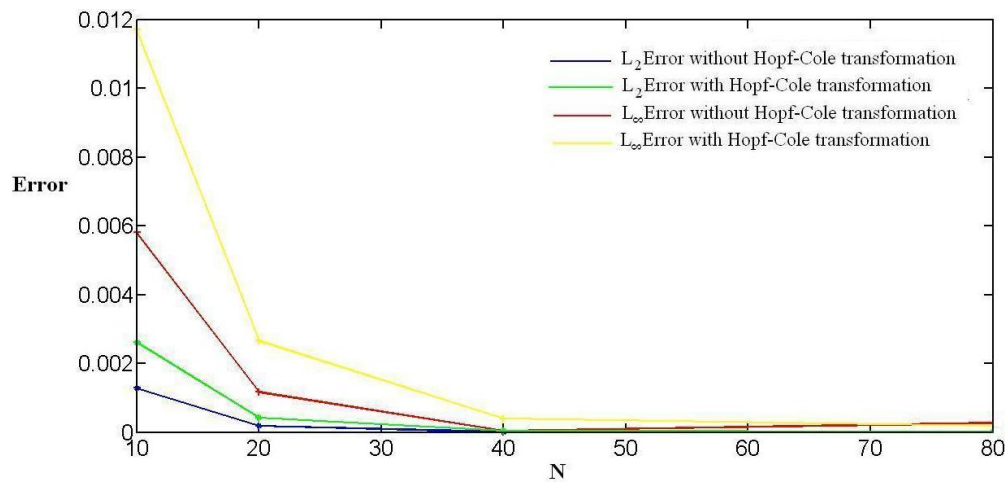

(a)

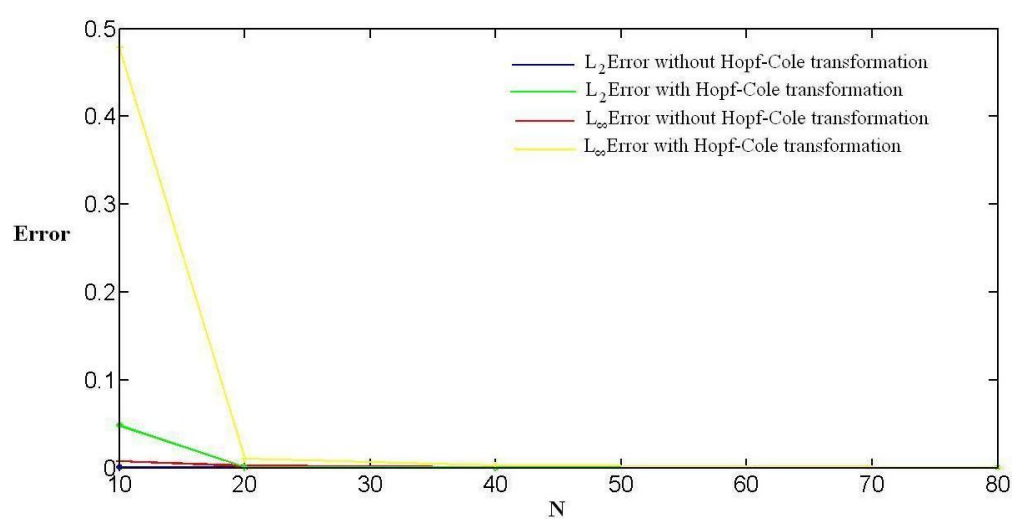

(b)

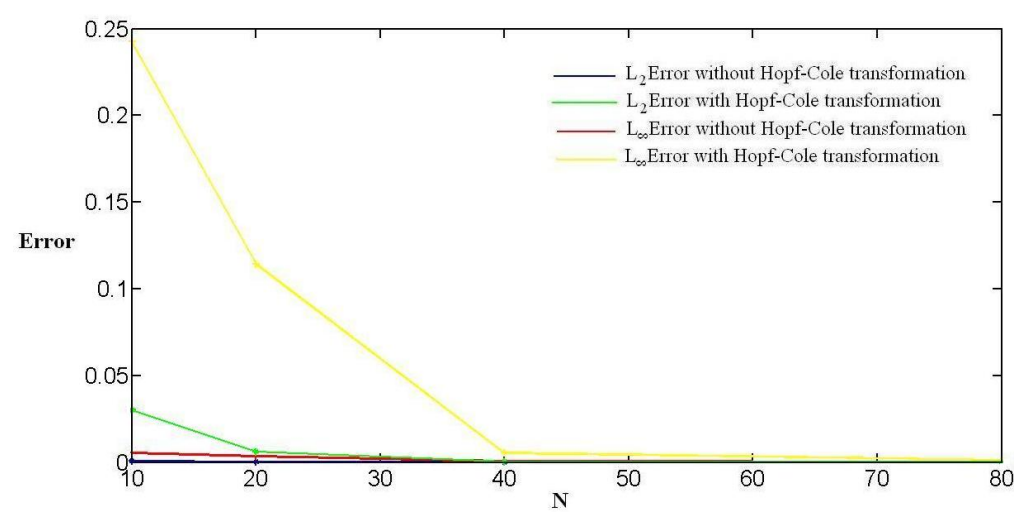

(c) 
Figure $4 L_{2}$ and $L_{\infty}$ error of ex. 1 at (a) $\tau=0.1$ for $v=1$ and $\Delta t=0.0001$ (b) $\tau=5$ for $v=0.01$ and $\Delta t=0.01$ (c) $\tau=7$ for $v=0.005$ and $\Delta t=0.01$ (d) $\tau=1$ for $v=0.1$ and $\Delta t=0.0001$ (continued) (see online version for colours)

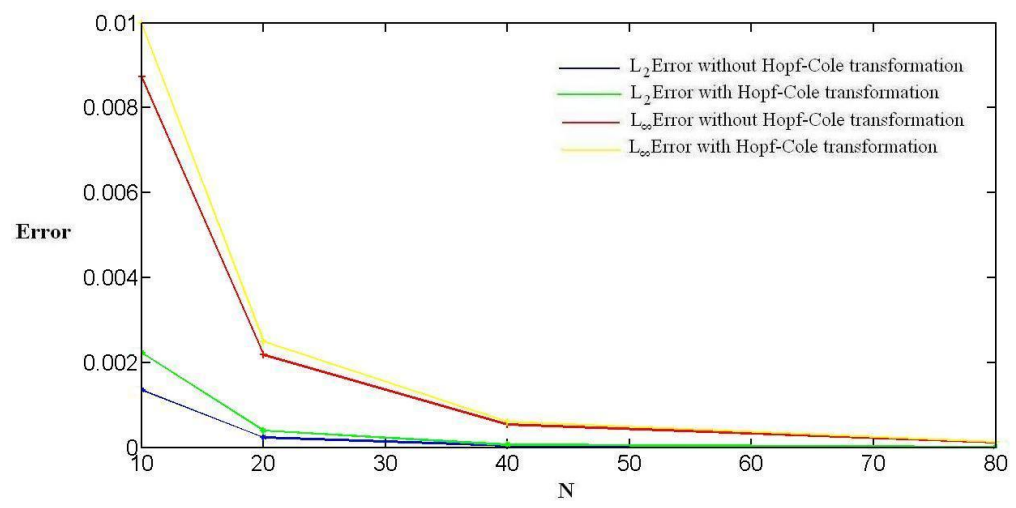

(d)

Figure $5 L_{2}$ and $L_{\infty}$ error of ex. 2 at (a) $\tau=1$ for $v=0.1$ and $\Delta t=0.0001$ (b) $\tau=0.1$ for $v=1$ and $\Delta t=0.0001$ (c) $\tau=5$ for $v=0.01$ and $\Delta t=0.01$ (d) $\tau=7$ for $v=0.005$ and $\Delta t=0.01$ (see online version for colours)

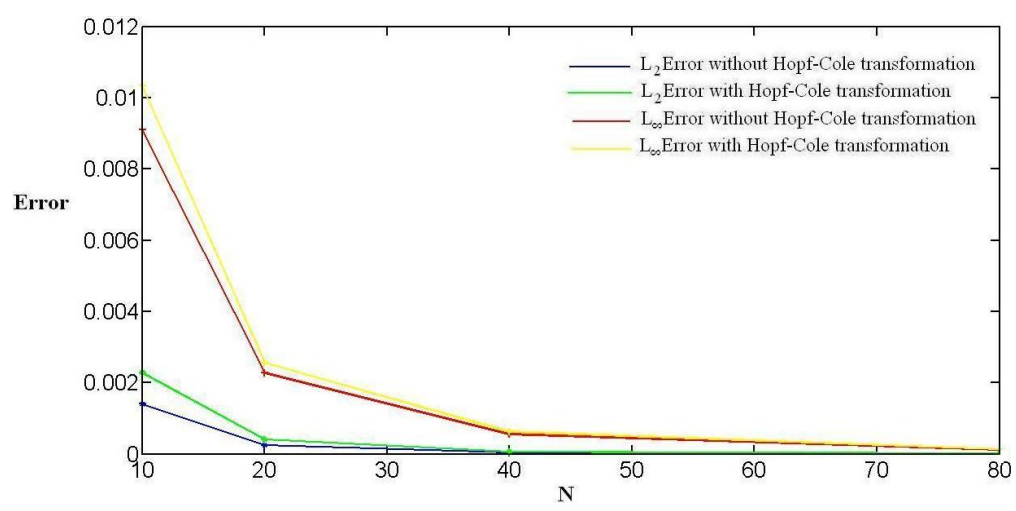

(a)

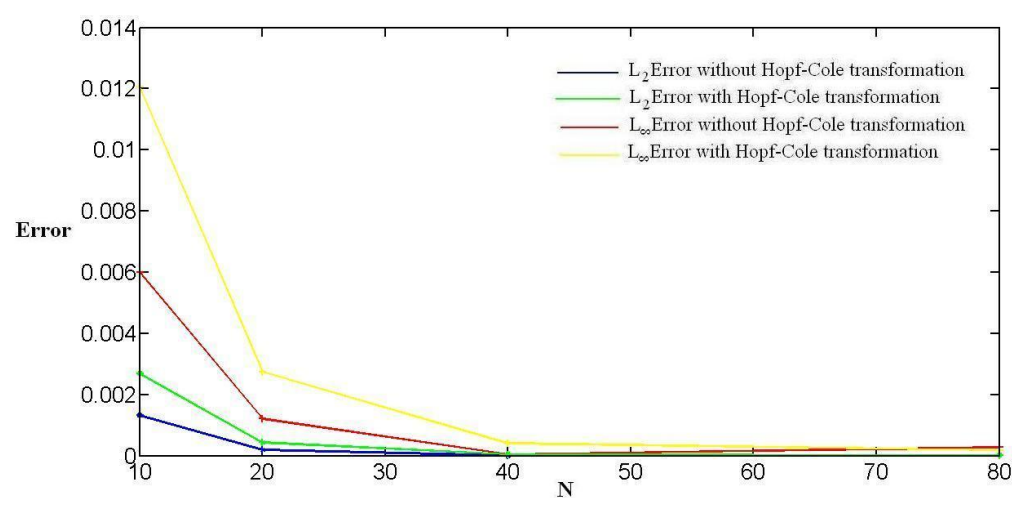

(b) 
Figure $5 L_{2}$ and $L_{\infty}$ error of ex. 2 at (a) $\tau=1$ for $v=0.1$ and $\Delta t=0.0001$ (b) $\tau=0.1$ for $v=1$ and $\Delta t=0.0001$ (c) $\tau=5$ for $v=0.01$ and $\Delta t=0.01$ (d) $\tau=7$ for $v=0.005$ and $\Delta t=0.01$ (continued) (see online version for colours)

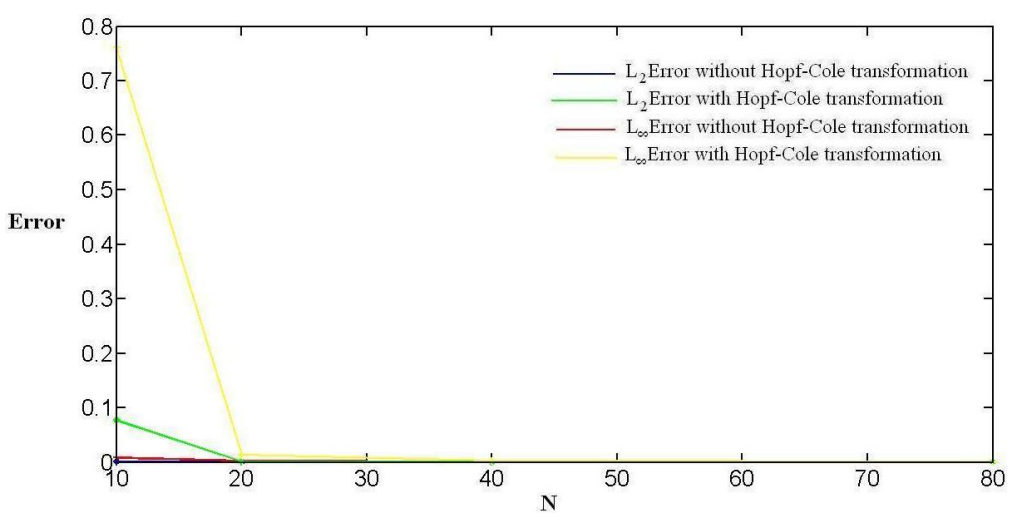

(c)

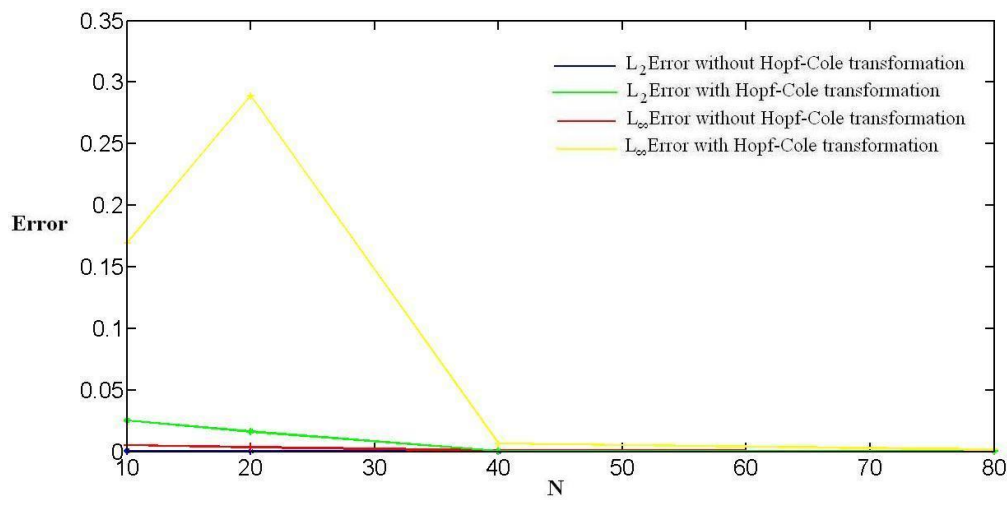

(d)

Table 9 Comparison of the numerical solution with the exact solution at different space points of ex. 1 at $\tau=0.1$ for $v=1$ and $\Delta t=0.0001$

\begin{tabular}{|c|c|c|c|c|c|}
\hline \multirow{2}{*}{$x$} & \multicolumn{2}{|c|}{$N=40$} & \multicolumn{2}{|c|}{$N=80$} & \multirow{2}{*}{$\begin{array}{c}\text { Exact } \\
\text { solution }\end{array}$} \\
\hline & With Hopf-Cole & Without Hopf-Cole & With Hopf-Cole & Without Hopf-Cole & \\
\hline 0.1 & 0.10940 & 0.10955 & 0.10958 & 0.10961 & 0.10953 \\
\hline 0.2 & 0.20954 & 0.20981 & 0.20988 & 0.20994 & 0.20979 \\
\hline 0.3 & 0.29156 & 0.29191 & 0.29202 & 0.29211 & 0.29189 \\
\hline 0.4 & 0.34754 & 0.34793 & 0.34808 & 0.34817 & 0.34792 \\
\hline 0.5 & 0.37118 & 0.37156 & 0.37175 & 0.37184 & 0.37157 \\
\hline 0.6 & 0.35868 & 0.35901 & 0.35922 & 0.35930 & 0.35904 \\
\hline 0.7 & 0.30960 & 0.30986 & 0.31006 & 0.31012 & 0.30990 \\
\hline 0.8 & 0.22760 & 0.22777 & 0.22793 & 0.22798 & 0.22781 \\
\hline 0.9 & 0.12057 & 0.12066 & 0.12075 & 0.12077 & 0.12068 \\
\hline
\end{tabular}


Table 10 Comparison of the numerical solution with the exact solution at different space points of ex. 1 at $\tau=0.01$ for $v=10$ and $\Delta t=0.00001$

\begin{tabular}{|c|c|c|c|c|c|}
\hline \multirow{2}{*}{$x$} & \multicolumn{2}{|c|}{$N=40$} & \multicolumn{2}{|c|}{$N=80$} & \multirow{2}{*}{$\begin{array}{c}\text { Exact } \\
\text { solution }\end{array}$} \\
\hline & With Hopf-Cole & Without Hopf-Cole & With Hopf-Cole & Without Hopf-Cole & \\
\hline 0.1 & 0.11449 & 0.11461 & 0.11466 & 0.11469 & 0.11461 \\
\hline 0.2 & 0.21793 & 0.21816 & 0.21826 & 0.21832 & 0.21816 \\
\hline 0.3 & 0.30029 & 0.30061 & 0.30075 & 0.30083 & 0.30061 \\
\hline 0.4 & 0.35352 & 0.35388 & 0.35406 & 0.35415 & 0.35389 \\
\hline 0.5 & 0.37230 & 0.37268 & 0.37287 & 0.37296 & 0.37269 \\
\hline 0.6 & 0.35464 & 0.35500 & 0.35518 & 0.35527 & 0.35501 \\
\hline 0.7 & 0.30210 & 0.30241 & 0.30257 & 0.30264 & 0.30242 \\
\hline 0.8 & 0.21974 & 0.21996 & 0.22008 & 0.22013 & 0.21997 \\
\hline 0.9 & 0.11561 & 0.11572 & 0.11578 & 0.11581 & 0.11573 \\
\hline
\end{tabular}

Table 11 Comparison of the numerical solution with the exact solution at different space points of ex. 2 at $\tau=0.1$ for $v=1$ and $\Delta t=0.0001$

\begin{tabular}{|c|c|c|c|c|c|}
\hline \multirow{2}{*}{$x$} & \multicolumn{2}{|c|}{$N=40$} & \multicolumn{2}{|c|}{$N=80$} & \multirow{2}{*}{$\begin{array}{c}\text { Exact } \\
\text { solution }\end{array}$} \\
\hline & With Hopf-Cole & Without Hopf-Cole & With Hopf-Cole & Without Hopf-Cole & \\
\hline 0.1 & 0.11275 & 0.11290 & 0.11293 & 0.11297 & 0.11289 \\
\hline 0.2 & 0.21600 & 0.21627 & 0.21634 & 0.21641 & 0.21625 \\
\hline 0.3 & 0.30062 & 0.30098 & 0.30109 & 0.30118 & 0.30097 \\
\hline 0.4 & 0.35846 & 0.35887 & 0.35902 & 0.35912 & 0.35886 \\
\hline 0.5 & 0.38301 & 0.38341 & 0.38360 & 0.38370 & 0.38342 \\
\hline 0.6 & 0.37028 & 0.37063 & 0.37084 & 0.37092 & 0.37066 \\
\hline 0.7 & 0.31975 & 0.32002 & 0.32022 & 0.32029 & 0.32007 \\
\hline 0.8 & 0.23514 & 0.23533 & 0.23549 & 0.23554 & 0.23537 \\
\hline 0.9 & 0.12460 & 0.12469 & 0.12478 & 0.12480 & 0.12472 \\
\hline
\end{tabular}

Table 12 Comparison of the numerical solution with the exact solution at different space points of ex. 2 at $\tau=0.01$ for $v=10$ and $\Delta t=0.00001$

\begin{tabular}{|c|c|c|c|c|c|}
\hline \multirow{2}{*}{$x$} & \multicolumn{2}{|c|}{$N=40$} & \multicolumn{2}{|c|}{$N=80$} & \multirow{2}{*}{$\begin{array}{c}\text { Exact } \\
\text { solution }\end{array}$} \\
\hline & With Hopf-Cole & Without Hopf-Cole & With Hopf-Cole & Without Hopf-Cole & \\
\hline 0.1 & 0.11814 & 0.11827 & 0.11833 & 0.11836 & 0.11827 \\
\hline 0.2 & 0.22489 & 0.22513 & 0.22524 & 0.22530 & 0.22513 \\
\hline 0.3 & 0.30989 & 0.31021 & 0.31037 & 0.31045 & 0.31022 \\
\hline 0.4 & 0.36483 & 0.36521 & 0.36539 & 0.36548 & 0.36521 \\
\hline 0.5 & 0.38422 & 0.38462 & 0.38481 & 0.38491 & 0.38463 \\
\hline 0.6 & 0.36602 & 0.36639 & 0.36658 & 0.36667 & 0.36640 \\
\hline 0.7 & 0.31181 & 0.31213 & 0.31229 & 0.31237 & 0.31214 \\
\hline 0.8 & 0.22681 & 0.22704 & 0.22716 & 0.22722 & 0.22705 \\
\hline 0.9 & 0.11933 & 0.11945 & 0.11951 & 0.11954 & 0.11946 \\
\hline
\end{tabular}


Figure 6 Numerical solution profiles of ex. 1 with Hopf-Cole transformation at $\Delta x=0.025$ for (a) $v=0.01, \Delta t=0.001$ (b) $v=0.005, \Delta \mathrm{t}=0.1$ (see online version for colours)

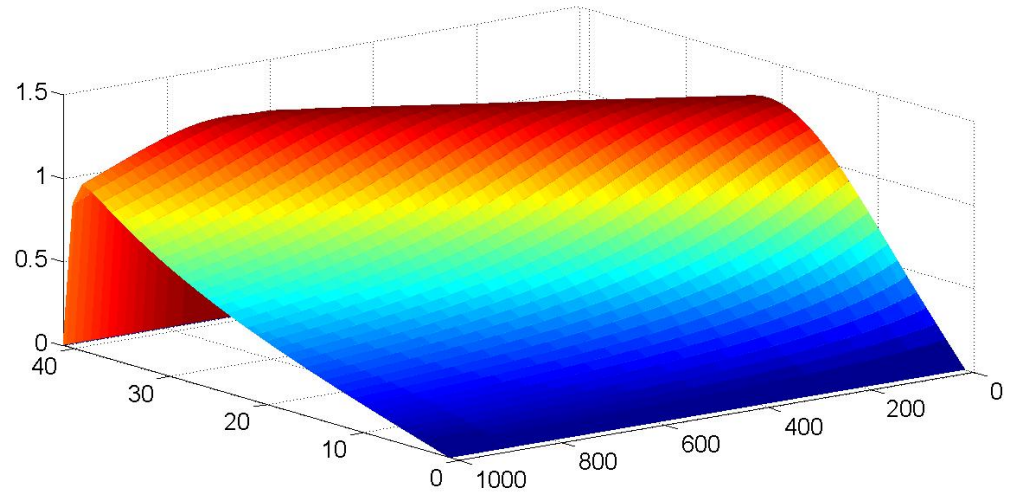

(a)

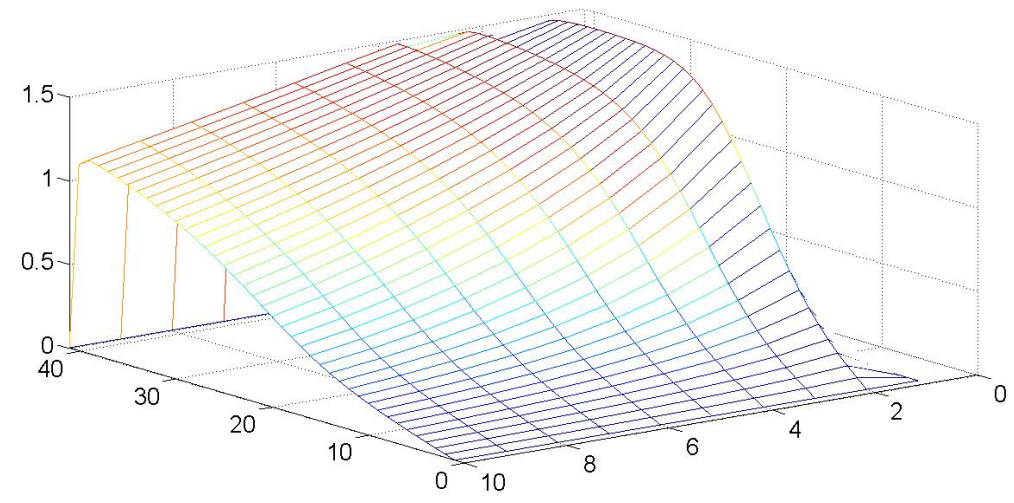

(b)

Figure 7 Numerical solution profiles of ex. 1 without Hopf-Cole transformation at $\Delta x=0.025$ for (a) $v=0.01, \Delta t=0.001$ (b) $v=0.005, \Delta t=0.1$ (see online version for colours)

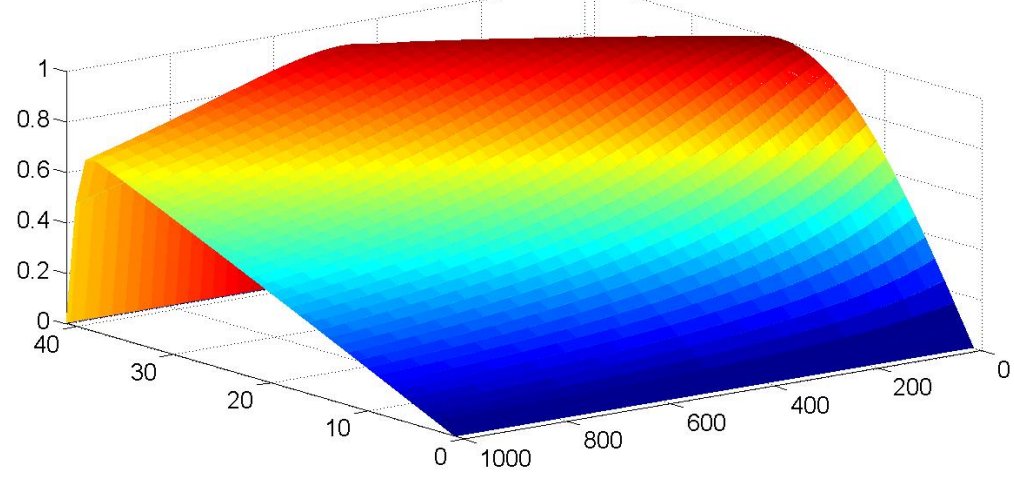

(a) 
Figure 7 Numerical solution profiles of ex. 1 without Hopf-Cole transformation at $\Delta x=0.025$ for (a) $v=0.01, \Delta t=0.001$ (b) $v=0.005, \Delta t=0.1$ (continued) (see online version for colours)

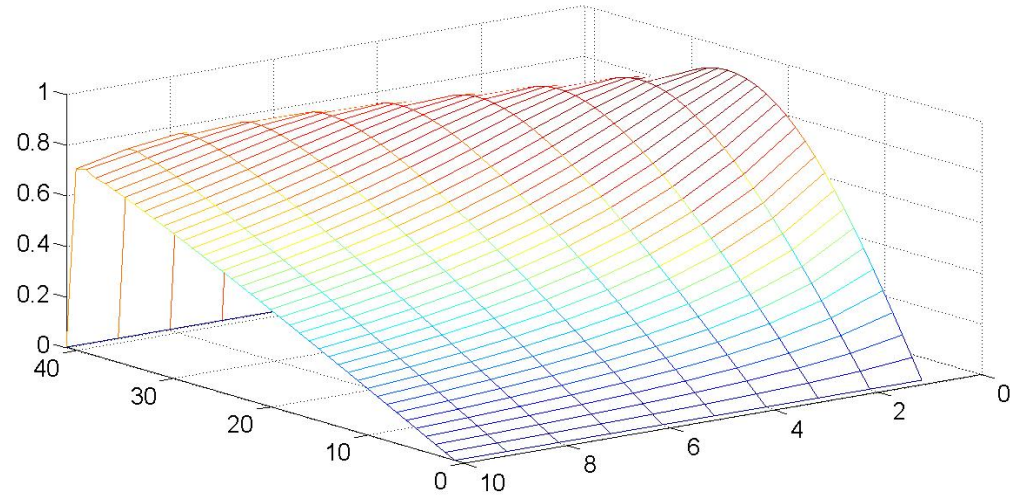

(b)

Figure 8 Numerical solution profiles of ex. 2 with Hopf-Cole transformation at $\Delta x=0.025$ for (a) $v=1, \Delta t=0.0001$ (b) $v=0.1, \Delta t=0.001$ (see online version for colours)

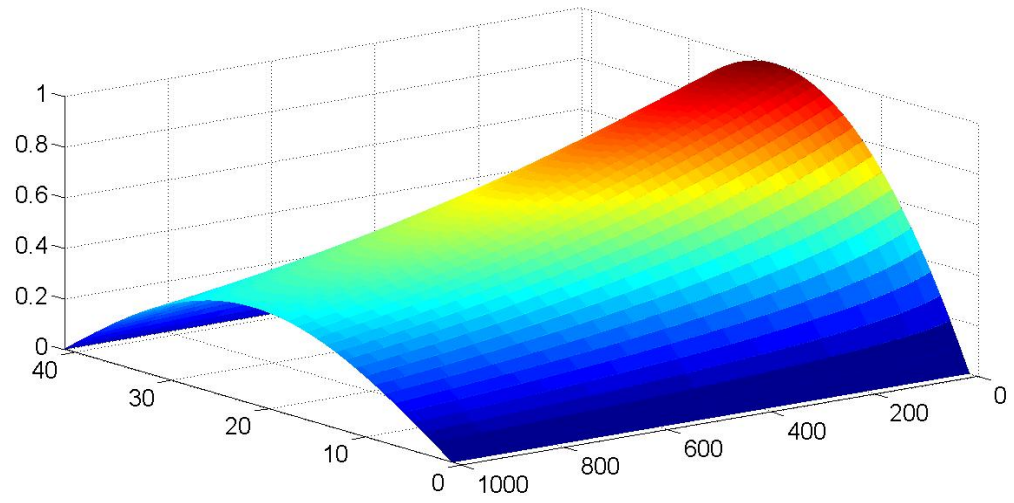

(a)

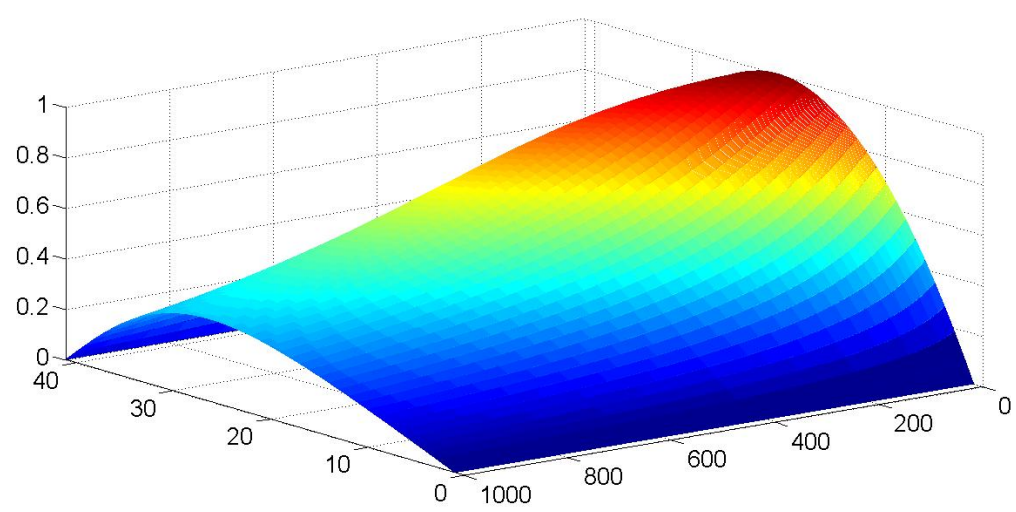

(b) 
Figure 9 Numerical solution profiles of ex. 2 without Hopf-Cole transformation at $\Delta x=0.025$ for (a) $v=1, \Delta \mathrm{t}=0.0001$ (b) $v=0.1, \Delta t=0.001$ (see online version for colours)

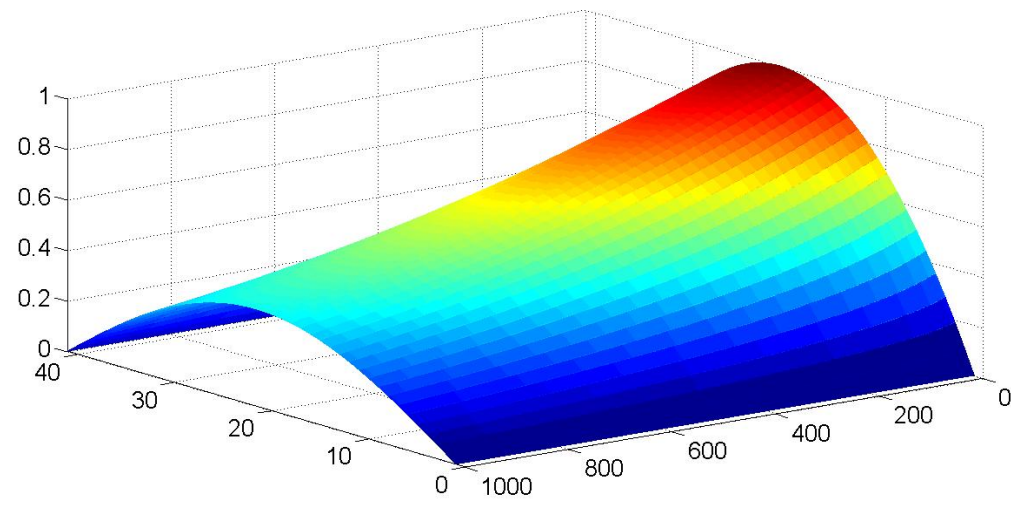

(a)

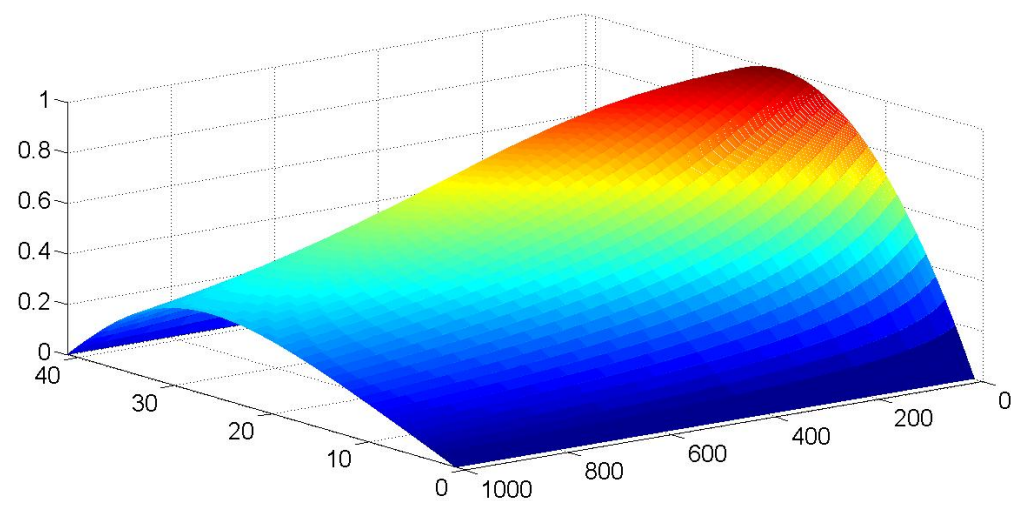

(b)

Computed results for both the schemes are illustrated in Tables 9 and 10 for test example 1. In Table 9, numerical solution computed with and without Hopf-Cole transformation is compared with the exact solution for $v=1$ and $\Delta t=0.0001$ at different space points. Table 10 includes comparison of numerical solution with the exact solution for $v=10$ and $\Delta t=0.00001$ at different space points. For test example 2, numerical solutions calculated by using both the schemes are shown in Tables 11 and 12. In Table 11, numerical solution computed with and without Hopf-Cole transformation is compared with exact solution for $v=1$ and $\Delta t=0.0001$. Whereas in Table 12, numerical solutions is compared with exact solution for $v=10$ and $\Delta \mathrm{t}=0.00001$. From Tables 9 to 12 , it is observed that the numerical solutions are in good agreement with exact solutions. These results show that the scheme is consistent and accurate of order two in both space and time. In order to show the physical behaviour of the given problem, surface plots of the computed solutions are shown in Figures 6 to 9 for distinct values of $v$ and $\Delta t$. Figures 6 and 7 show the numerical solution profiles of example 1 at $\Delta x=0.025$. Identical values of $v(v=0.005$ and $v=0.01)$ are taken to compare the behaviour of velocity distribution obtain with and without Hopf-Cole transformation. For example 2, surface plots are given in Figures 8 and 9 for $\Delta x=0.025$. Numerical solution profile of 
example 2 with Hopf-Cole transformation is plotted in Figure 8 for $v=0.1$ and $v=1$. For the same value of $v$, surface plot of example 2 without Hopf-Cole transformation is given in Figure 9. This clearly gives an indication that the velocity distribution is time dependent.

\section{Conclusions}

The proposed implicit scheme without Hopf-Cole transformation and the modified box scheme coupled with Hopf-Cole transformation are used to study the properties of the solution of Burgers' equation for the wide range of kinematic viscosity $(v)$. The present numerical experiments have confirmed that, the proposed implicit scheme without Hopf-Cole transformation is more accurate than the modified box scheme coupled with Hopf-Cole transformation. It is concluded from the Figures 4 and 5 that, Hopf-Cole trans-formation negatively affect performance of the difference scheme. $L_{2}$ and $L_{\infty}$ error of numerical solution obtained without Hopf-Cole transformation is less. Both the schemes are second order accurate in space and time. There is no requirement with respect to mesh size restriction. Tables 9 to 12 indicate that the results are in good agreement with the exact solution for modest values of $v$. The physical behaviour of the solution is explained in Figures 6 to 9 and it is concluded that the numerically calculated values are in close agreement with the exact solution.

\section{Acknowledgements}

The authors thank the anonymous referees for their valuable time, effort and extensive comments which help to improve the quality of the paper.

\section{References}

Bakodah, H.O. (2011) 'Non-central 7-point formula in the method of lines for parabolic and Burgers' equations', IJRRAS, Vol. 8, No. 3, pp.328-336.

Bateman, H. (1915) 'Some recent researches on the motion of fluids', Monthly Weather Rev., Vol. 43, pp.163-170.

Benton, E.R. and Platzman, G.W. (1972) 'A table of solutions of the one-dimensional Burgers' equations', Quart. Appl. Math., Vol. 30, No. 2, pp.195-212.

Bonkile, M.P., Awasthi, A. and Jayaraj, S. (2015) 'A numerical simulation based on modified Keller box scheme for fluid flow: the unsteady viscous Burgers' equation', Mathematical Analysis and its Applications, Springer Proceedings in Mathematics and Statistics, Vol. 143, pp.565-575.

Brander, O. and Hedenfalk, J. (1998) 'A new formulation of the general solution to burgers equation', Wave Motion, Vol. 28, No. 4, pp.319-332.

Burgers, J.M. (1939) 'Mathematical examples illustrating relations occurring in the theory of turbulent fluid motion', Transactions on Royal Netherlands Academic Science, Vol. 17, pp.1-53, Amsterdam.

Burgers, J.M. (1948) 'A mathematical model illustrating the theory of turbulence', Advances in Applied Mechanics, Vol. 1, pp.171-199. 
Burns, J., Balogh, A., Gilliam, D. and Shubol, V. (1998) 'Numerical stationary solutions for a viscous Burgers' equation', Journal of Mathematical Systems, Estimation, and Control, Vol. 8, pp.1-16.

Cole, J.D. (1951) 'On a quaslinear parabolic equations occurring in aerodynamics', Quart. Appl. Math., Vol. 9, No. 2, pp.225-236.

Collier, N., Radwan, H., Dalcin, L. and Calo, V. (2013) 'Time adaptivity in the diffusive wave approximation to the shallow water equations', Journal of Computer Science, Vol. 4, No. 3, pp.152-156.

Dhawan, S., Kapoor, S., Kumara, S. and Rawat, S. (2012) 'Contemporary review of techniques for the solution of nonlinear burgers equation', Journal of Computational Science, Vol. 3, No. 5, pp.405-419.

Gulsu, M. (2006) 'A finite difference approach for solution of Burgers' equation', Applied Mathematics and Computation, Vol. 175, No. 2, pp.1245-1255.

Hopf, E. (1950) 'The partial differential equation $u_{t}+u u_{x}=v u_{x x}$ ', Comm. Pure Appl. Math., Vol. 3, pp.201-230.

Kadalbajoo, M. and Awasthi, A. (2006) 'A numerical method based on Crank-Nicolson scheme for Burgers' equation', Appl. Math. Comput., Vol. 182, No. 2, pp.1430-1442.

Kardar, M., Parisi, G. and Zhang, Y. (1986) 'Dynamical scaling of growing interfaces', Physical Review Letters, Vol. 56, No. 9, pp.889-892.

Keller, H.B. (1970) 'A new difference scheme for parabolic problems', Numerical Solutions of Partial Difference Equations, Vol. 2, pp.327-350.

Kurtz, L.A., Smith, R.E., Parks, C.L. and Boney, L.R. (1977) 'A comparison of the method of lines to finite difference techniques in solving time-dependent partial differential equations', Computers and Fluids, Vol. 6, No. 2, pp.49-70.

Kutluay, S., Bahadir, A. and Ozdes, A. (1999) 'Numerical solution of one-dimensional burgers equation: explicit and exact-explicit finite difference methods', Journal of Computational and Applied Mathematics, Vol. 103, No. 2, pp.251-261.

Molchanov, S., Surgailis, D. and Woyczynski, W. (1997) 'The large-scale structure of the universe and quasi-voronoi tessellation of shock fronts in forced burgers turbulence in rn', Annals of Applied Probability, Vol. 7, No. 1, pp.200-228.

Molchanov, S., Surgailis, D. and Woyczynskii, W. (1995) 'Hyperbolic asymptotics in Burgers turbulence', Communications in Mathematical Physics, Vol. 168, No. 1, pp.209-226.

Nieuwstadt, F.T. and Steketee, J.A. (1995) Selected Papers of J.M. Burgers, Springer Science+Business Media, B.V., Dordrecht.

Oymak, O. and Selcuk, N. (1996) 'Method-of-lines solution of time-dependent two-dimensional Navier-Stokes equations', International Journal for Numerical Methods in Fluids, Vol. 23, No. 5, pp.455-466.

Rodin, E.Y. (1970) 'On some approximate and exact solutions of boundary value problems for burgers equation', Journal of Mathematical Analysis and Application, Vol. 30, No. 2, pp.401-414.

Rothe, E. (1930) 'Zweidimensionale parabolisehe randwertaufgaben als grenzfall eindimensionaler randwertaufgaben', Mathematische Annalen, Vol. 102, No. 1, pp.650-670.

Shampine, L.F. (1994) 'Ode solvers and the method of lines', Numerical Methods for Partial Differential Equations, Vol. 10, pp.739-755.

Shandarin, S. and Zeldovich, Y. (1989) 'Turbulence, intermittency, structures in a self-gravitating medium: the large-scale structure of the universe', Reviews of Modern Physics, Vol. 61, No. 2, pp.185-220. 
Shu, C.W. (1988) 'Total-variation-diminishing time discretizations', SIAM J. Sci. Statist. Comput., Vol. 9, No. 6, pp.1073-1084.

Spiteri, R. and Ruuth, S.J. (2002) 'A new class of optimal high-order strong-stability-preserving time discretization methods', SIAM J. Numer. Anal., Vol. 40, No. 2, pp.469-491.

Vergassola, M., Dubrulle, B., Frisch, U. and Noullez, A. (1994) 'Burgers equation, devil's staircases and mass distribution for the large scale structure', Astronomy and Astrophysics, Vol. 289, No. 2, pp.325-356. 